\title{
Density perturbations in the Ekpyrotic Universe and string-inspired generalizations
}

\author{
Shinji Tsujikawa \\ Research Center for the Early Universe, University of Tokyo, Hongo, Bunkyo-ku, Tokyo 113-0033, Japan
}

(October 30, 2018)

\begin{abstract}
We study density perturbations in several cosmological models motivated by string theory. The evolution and the spectra of curvature perturbations $\mathcal{R}$ are analyzed in the Ekpyrotic scenario and nonsingular string cosmologies. We find that these string-inspired models generally exhibit blue spectra in contrast to standard slow-roll inflationary scenarios. We also clarify the parameter range where $\mathcal{R}$ is enhanced on superhorizon scales.
\end{abstract}

\section{INTRODUCTION}

The pre-big-bang (PBB) cosmological models based on the low-energy effective actions of string theory have been widely studied as possible candidates to describe the evolution of the Universe at very high density [1]. In the PBB scenario there exist two disconnected branches, one of which corresponds to the stage of superinflation driven by the kinetic term of the dilaton field and another of which is the Friedmann branch of decelerating expansion [2.3. . While it is difficult to avoid the big bang singularity in the tree-level string action $4,5,5]$, two branches can be smoothly joined to each other by taking into account quantum loop corrections.

Among the nonsingular string-inspired scenarios, the model proposed by Antoniadis et al. [6] involves the Gauss-Bonnet curvature invariant coupled to the modulus field, which enables the big bang singularity to be avoided even in the closed [7] and the anisotropic Bianchi type-I Universes [8]. In the presence of the higher-order derivatives and the Gauss-Bonnet term coupled to the dilaton field, it was found in refs. [9, 10] that the prebig-bang inflationary evolution is followed by a phase with decreasing curvature without a singularity (see also [11-13). Recently a new scenario -the Ekpyrotic model - has been proposed by Khoury et al. 114,15]. According to this scenario, the collision of two parallel branes imbedded in the extra-dimensional bulk signals the beginning of the hot, expanding, big bang of the standard cosmology. Prior to the collision the Universe was slowly contracting.

It should be possible to discriminate the viability of these models by evaluating the spectra of density perturbations. In the PBB scenario without loop corrections, the curvature perturbation $\mathcal{R}$ is blue-tilted with spectral index $n \simeq 4$ [16], which contradicts with the observationally supported flat spectra with $n \simeq 1$. In order to make contact with observations precisely, it is very important to extend to nonsingular cosmological models with a successful graceful exit. In what follows we will analyze the spectra of curvature perturbations in the nonsingular models and the Ekpyrotic scenario. We will also clarify the cases where $\mathcal{R}$ is amplified on superhorizon scales even in the one-field model. This should again be contrasted with standard single-field inflationary models where $\mathcal{R}$ is constant on large scales.

\section{PERTURBED EQUATIONS}

Let us consider the following four-dimensional action

$$
\begin{aligned}
& S=\int d^{4} x \sqrt{-g}\left[\frac{1}{2} f(R, \phi)-\frac{1}{2} \omega(\phi)(\nabla \phi)^{2}\right. \\
& \left.-V(\phi)+\mathcal{L}_{c}\right],
\end{aligned}
$$

where $f(R, \phi)$ is a function of the Ricci scalar $R$ and a scalar field $\phi . \omega(\phi)$ and $V(\phi)$ are general functions of $\phi$. The Lagrangian $\mathcal{L}_{c}$ represents the higher-order corrections to the tree-level action. The action (2.1) includes a wide variety of theories, e.g., Einstein gravity, scalar tensor theories, and the low-energy effective string theories reduced from the higher-dimensional actions. In what follows we shall focus on the models inspired from string theories.

While it is difficult to avoid the big bang singularity in the PBB scenario using only the tree-level action, the existence of loop corrections is capable to overcome such singularity problem. In this work we shall consider the higher-order corrections, given by

$$
\mathcal{L}_{c}=-\frac{1}{2} \alpha^{\prime} \lambda \xi(\phi)\left[c R_{\mathrm{GB}}^{2}+d(\nabla \phi)^{4}\right]
$$

where $R_{\mathrm{GB}}^{2}=R^{2}-4 R^{\mu \nu} R_{\mu \nu}+R^{\mu \nu \alpha \beta} R_{\mu \nu \alpha \beta}$ is the GaussBonnet term. Hereafter the inverse string tension, $\alpha^{\prime}$, is set to unity.

A perturbed space-time metric has the following form for scalar perturbations in an arbitrary gauge:

$$
\begin{aligned}
d s^{2}= & -(1+2 A) d t^{2}+2 a(t) B_{, i} d x^{i} d t \\
& +a^{2}(t)\left[(1-2 \psi) \delta_{i j}+2 E_{, i, j}\right] d x^{i} d x^{j},
\end{aligned}
$$

where $a(t)$ is the scale factor, and a comma means usual flat space coordinate derivative. It is convenient to introduce gauge-invariant variable, defined by

$$
\mathcal{R} \equiv \psi+\frac{H}{\dot{\phi}} \delta \phi,
$$


where $H \equiv \dot{a} / a$ is the Hubble expansion rate. This quantity corresponds to the curvature perturbation in the uniform curvature gauge. The perturbed Einstein equations for scalar perturbations are written in the form [17. 18]

$$
\frac{1}{a^{3} Q}\left(a^{3} Q \mathcal{R}\right)^{\bullet}-s \frac{\Delta}{a^{2}} \mathcal{R}=0,
$$

where

$$
\begin{gathered}
Q \equiv \frac{\omega \dot{\phi}^{2}+3 I\left(\dot{F}-4 \lambda c \dot{\xi} H^{2}\right)-6 \lambda d \xi \dot{\phi}^{4}}{(H+I)^{2}}, \\
s \equiv 1+\frac{4 \lambda c \xi \dot{\phi}^{4}-16 \lambda c \dot{\xi} \dot{H} I+8 \lambda c(\ddot{\xi}-\dot{\xi} H) I^{2}}{\omega \dot{\phi}^{2}+3 I\left(\dot{F}-4 \lambda c \dot{\xi} H^{2}\right)-6 \lambda d \xi \dot{\phi}^{4}} .
\end{gathered}
$$

Here $F$ and $I$ are defined as $F=\partial f / \partial R$ and $I \equiv(\dot{F}-$ $\left.4 \lambda c \dot{\xi} H^{2}\right) /(2 F-8 \lambda c \dot{\xi} H)$.

Let us introduce a new quantity, $\Psi \equiv z \mathcal{R}$, with $z \equiv$ $a \sqrt{Q}$. Then the each Fourier component of $\Psi$ satisfies the second order differential equation

$$
\Psi_{k}^{\prime \prime}+\left(s k^{2}-\frac{z^{\prime \prime}}{z}\right) \Psi_{k}=0
$$

where a prime denotes the derivative with respect to conformal time, $\eta=\int a^{-1} d t$. In the large scale limit, $\left|s k^{2}\right| \ll\left|z^{\prime \prime}\right| z \mid$, eq. (2.8) is easily integrated to give

$$
\mathcal{R}_{k}=C_{k}+D_{k} \int \frac{d \eta}{z^{2}}
$$

where $C_{k}$ and $D_{k}$ are integration constants corresponding to the growing and decaying mode, respectively. The curvature perturbation is conserved on superhorizon scales as long as the decaying mode is not strongly dominating, as in the case of the single field, slow-roll inflationary scenarios. A counter example was recently found in ref. 19] in the context of fast-roll inflation.

In the string-inspired models, when the evolution of $z$ before the bounce is given in the form

$$
z \propto(-\eta)^{\alpha}
$$

the second term in eq. (2.9) yields $\int d \eta / z^{2} \propto(-\eta)^{1-2 \alpha}$. Therefore $\mathcal{R}_{k}$ can be amplified for $\alpha \geq 1 / 2$ on superhorizon scales, while it is not for $\alpha<1 / 2$ (Note that $\mathcal{R}_{k} \propto \ln (-\eta)$ for $\left.\alpha=1 / 2\right)$. Whether this enhancement occurs or not depends on the models of string theory as we will show later.

In order to obtain the spectra of curvature perturbations, it is required to go to the next order solution of eq. (2.8). If $s$ is a positive constant, the solution for $\Psi_{k}$ is expressed by the combination of the Hankel functions:

$$
\Psi_{k}=\frac{\sqrt{-\pi \eta}}{2}\left[c_{1} H_{\nu}^{(1)}(x)+c_{2} H_{\nu}^{(2)}(x)\right],
$$

where $x \equiv-\sqrt{s} k \eta$ and $\nu \equiv|1-2 \alpha| / 2$. Making use of the relation $H_{\nu}^{(2,1)}(-\sqrt{s} k \eta) \rightarrow \pm(i / \pi) \Gamma(\nu)(-\sqrt{s} k \eta / 2)^{-\nu}$ for long wavelength perturbations $(-\sqrt{s} k \eta \rightarrow 0)$, we get the spectrum of curvature perturbations as

$$
P_{\mathcal{R}} \equiv \frac{k^{3}}{2 \pi^{2}}\left|\mathcal{R}_{k}\right|^{2} \propto k^{3-2 \nu},
$$

in which case the spectral tilt is

$$
n-1=3-|1-2 \alpha| .
$$

If the corrections $\mathcal{L}_{c}$ are not taken into account, $s$ is exactly unity as in the case of the Einstein gravity. In the context of nonsingular string cosmologies, $s$ is generally the time-varying function and even can change sign due to the presence of the higher-order corrections. In such cases the formula (2.13) can not be directly applied, but it is still valid if $s$ is slowly varying positive function. In the next section we shall analyze the evolution and the spectra of curvature perturbations in several different models.

\section{STRING INSPIRED MODELS}

\section{A. Ekpyrotic Universe}

The Ekpyrotic scenario is based on the collision of two parallel branes imbedded in the extra-dimensional bulk. The position of the moving brane before the collision is represented by a scalar field $\phi$, with negative exponential potential

$$
V(\phi)=-V_{0} e^{-\sqrt{2 / p} \phi} .
$$

In the original version of the Ekpyrotic scenario [14], the collision of branes takes place at some finite value of $\phi$. The effective action in this model corresponds to $f=R$, $\omega=1, V \neq 0$, and $\mathcal{L}_{c}=0$, in which case one has $Q=$ $\dot{\phi}^{2} / H^{2}$ and $s=1$. The background evolution before the bounce $(t<0)$ is given by

$$
a \propto(-t)^{p}, \quad H=\frac{p}{t}, \quad \dot{\phi}^{2}=\frac{2 p}{t^{2}}, \quad V=\frac{p(3 p-1)}{t^{2}} .
$$

Therefore we find $z=a \dot{\phi} / H \propto(-t)^{p} \propto(-\eta)^{p /(1-p)}$, which means $\alpha=p /(1-p)$. Since a very slow contraction leads to the condition, $0<p \ll 1 / 3, \alpha$ is constrained to be $0<\alpha \ll 1 / 2$. Then the curvature perturbation is conserved on large scales due to the negligible contribution of the decaying mode of eq. (2.9).

Since $s$ is exactly unity in this case, the result (2.13) can be applied to give

$$
n-1=\frac{2}{1-p} .
$$

Therefore one has the blue spectrum $n \simeq 3$ for $p \simeq 0$ $(\alpha \simeq 0)$. This means the difficulty to generate the scaleinvariant spectrum in the old Ekpyrotic scenario, which supports the results in refs. 20 22. 
In the new Ekpyrotic scenario 15, it is assumed that the brane collision occurs at the field value $\phi=-\infty$ with vanishing potential $V \simeq 0$. This corresponds to the case with $p=1 / 3$ and $\alpha=1 / 2$, which indicates that $\mathcal{R}_{k}$ is logarithmically divergent at the bounce $(\eta=0)$. On the other hand the gravitational potential $\Phi_{k}$ is finite and even exhibits nearly flat spectra with small amplitudes [15]. In the case where $\mathcal{R}$ is divergent and $\Phi$ is much smaller than unity, however, we cannot choose any appropriate time-displacement $\delta t$ in gauge transformations [23. Therefore the cosmological perturbation theory is no longer valid at the bounce, which suggests the difficulty to produce the observationally supported curvature perturbations. This situation is similar to the tree-level PBB scenario where $\mathcal{R}_{k}$ grows logarithmically toward the singularity at $\eta=0$ and the spectrum of the curvature perturbation is far from scale-invariant.

\section{B. Modulus-driven inflation}

Let us next consider a model of $f=R, \omega=1, V=0$, $c=-1, d=0$, and $\xi=\ln \left[2 e^{\phi} \eta^{4}\left(i e^{\phi}\right)\right]$ with $\eta\left(i e^{\phi}\right)$ being the Dedekind $\eta$ function [6]. The singularity problem which plagues the PBB scenario can be avoided by taking into account the above form of the one-loop correction in the Einstein frame with modulus $(\phi)$ and dilaton fields. Since the evolution of the solution is mainly determined by the motion of the modulus field, we shall focus on the one-field system with positive $\lambda$ whereby singularity can be avoided.

Starting from a large initial value, $|\phi| \gg 1$, the Universe exhibits superinflation $(\dot{H}>0)$ until the graceful exit at $t=0$. During this stage, the background evolution is given by 8,24

$$
a \simeq a_{0}, \quad H=\frac{H_{0}}{t^{2}}, \quad \dot{\phi}=\frac{5}{(-t)},
$$

where $a_{0}$ and $H_{0}$ are constants. After the Hubble parameter reaches its peak value $H=H_{\max }$, the system connects to the Friedmann-like Universe with $H \simeq 1 /(3 t)$.

During the modulus-driven inflation, one has $\dot{\xi} \simeq$ $-(2 \pi / 3) \dot{\phi} \sin \mathrm{h} \phi \simeq(\pi / 3) \dot{\phi} e^{-\phi} \propto(-t)^{4}$. Therefore the value $Q$ defined by (2.6) evolves as $Q \propto(-t)^{2}$, which leads to $z \propto(-t) \propto(-\eta)$. Since $\alpha=1$ in this case, the curvature perturbation is enhanced on superhorizon scales during superinflation due to the growth of the second term in eq. (2.9). Even in the one-field model the conservation of $\mathcal{R}_{k}$ can be violated after horizon crossing when $\alpha$ is larger than $1 / 2$. We should keep in mind that the conservation of $\mathcal{R}_{k}$ is the specifics of the slowroll inflationary scenarios where the strongly dominated decaying mode is excluded (see refs. 19,25).

In the present model $s$ is much larger than unity during modulus-driven inflation and is proportional to $(-t)$, in which case eq. (2.13) is not applicable. From eq. (2.5) we easily find that $R_{k}$ satisfies

$$
\ddot{\mathcal{R}}_{k}+\frac{2}{t} \dot{\mathcal{R}}_{k}-\beta \frac{k^{2}}{a_{0}^{2}} t \mathcal{R}_{k}=0,
$$

where $\beta(>0)$ is a constant that depends on $H_{0}$. The solution of this equation is expressed in terms of the Bessel functions with $x \equiv \frac{2}{3} \sqrt{\beta} \frac{k}{a_{0}}(-t)^{3 / 2}$ :

$$
\mathcal{R}_{k}=(-t)^{-1 / 2}\left[c_{1} J_{-1 / 3}(x)+c_{2} J_{1 / 3}(x)\right],
$$

which asymptotically approaches the Minkowski vacuum for $x \rightarrow \infty$. Making use of the relation $J_{ \pm 1 / 3}(x) \propto k^{ \pm 1 / 3}$ in the $x \rightarrow 0$ limit, the spectrum of the long wave curvature perturbation is

$$
P_{\mathcal{R}} \propto k^{7 / 3}
$$

This is a blue spectrum with spectral index $n=10 / 3$. While the curvature perturbation grows on superhorizon scales, its spectrum is not scale invariant. In order to generate the scale invariant spectra, one requires the power exponent of $z$ close to $\alpha=2$ or $\alpha=-1$.

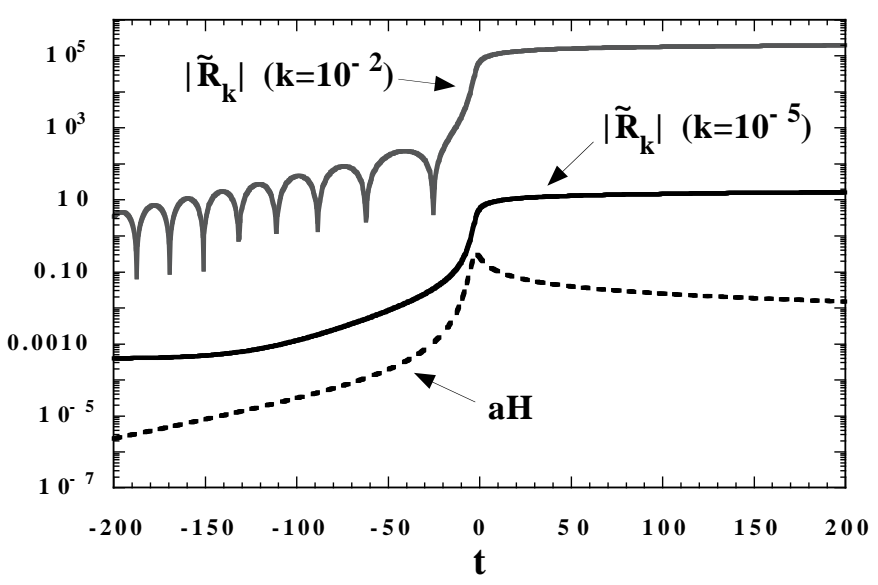

FIG. 1. The evolution of the quantity $a H$ and the curvature perturbation $\left|\tilde{\mathcal{R}}_{k}\right|=\left|k^{3 / 2} \mathcal{R}_{k}\right|$ for two different momenta during the modulus-driven inflation and the subsequent Friedmann-like Universe. We choose $\lambda=6 / \pi, \sigma=0$, and $\dot{\sigma}=0.2$ at $t=0$, whereby singularity is avoided.

In Fig. 11 we plot the evolution of the curvature perturbation for two modes $k=10^{-5}$ and $10^{-2}$. The mode $k=10^{-5}$ crosses the horizon $(k=a H)$ around $t=-150$, after which $\mathcal{R}_{k}$ continues to be enhanced until the graceful exit and freezes for $t>0$. The mode $k=10^{-2}$ stays inside the horizon until $t \lesssim-10$, during which the curvature perturbation exhibits some growth with oscillations $\mathcal{R}_{k} \propto(-t)^{-5 / 4} \cos (x)$ as found by eq. (3.6). After horizon crossing the evolution of $\mathcal{R}_{k}$ is similar to the $k=10^{-5}$ case.

It was claimed in ref. 26] that the bouncing Universe generally exhibits singular behavior of the gravitational potential $\Phi_{k}$. This result can not be directly applied to our model since the Universe is slowly expanding due to the presence of higher-order quantum corrections. In fact 
the curvature perturbation $\mathcal{R}_{k}$ as well as the background quantities are finite at the graceful exit, which means that the gravitational potential remains finite from eq. (2.4). Therefore the cosmological perturbation theory can be valid as long as the fluctuations do not exceed the linear level. This is contrasted with the new Ekpyrotic scenario where $\mathcal{R}_{k}$ is divergent at the bounce.

\section{Dilaton-driven inflation}

Nonsingular cosmological solutions have been also found in refs. [9 11] by including the higher order derivatives and loop corrections. The simplest version corresponds to the case with $f=e^{-\phi} R, \omega=\xi=-e^{-\phi}$, $V=0, c=-1, d=1$, and $\lambda=-1 / 4$, which we shall analyze below. Notice that the PBB scenario does not include the $\alpha^{\prime}$ correction in eq. (2.1), in which case the background evolution is described by $a \propto(-\eta)^{(1-\sqrt{3}) / 2}$ and $e^{\phi}=(-\eta)^{-\sqrt{3}}$ in the string frame [3]. Then one has $z \propto(-\eta)^{1 / 2}$, yielding the blue spectrum, $n=4$.

In the presence of the $\alpha^{\prime}$ correction, the initial lowcurvature dilaton-driven phase is followed by the string phase with linearly growing dilaton and nearly constant Hubble parameter (see Fig. 2). The fixed values during this stage are $\dot{\phi}_{f} \simeq 1.40$ and $H_{f} \simeq 0.62$, leading to the sufficient amount of inflation with e-folds $N \equiv \ln \left(a / a_{i}\right)>$ 60 provided that the dilaton field satisfies $|\phi| \gg 1$ initially [18]. In Fig. 2 inclusion of the loop corrections makes it possible to connect smoothly to the Friedmann branch around $N \simeq 60$.

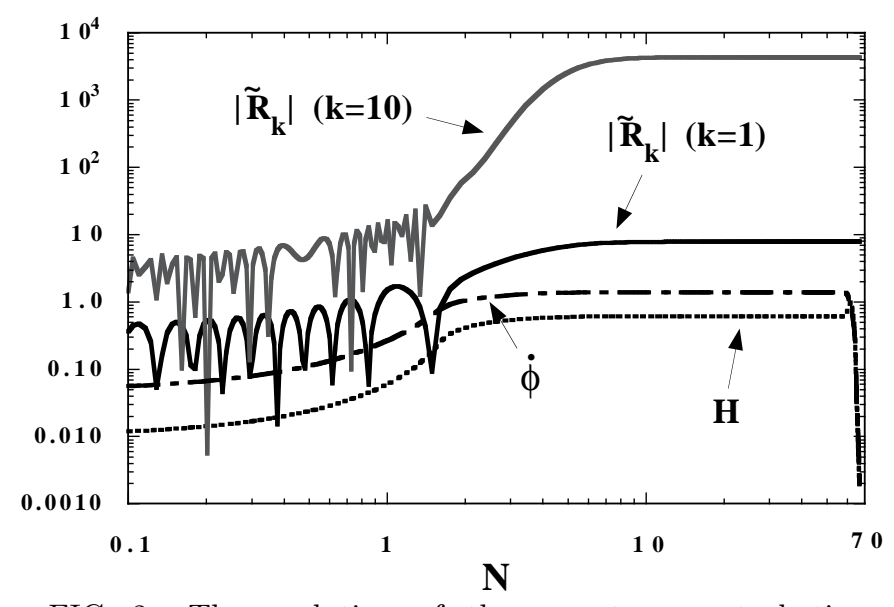

FIG. 2. The evolution of the curvature perturbation $\left|\tilde{\mathcal{R}}_{k}\right|=\left|k^{3 / 2} \mathcal{R}_{k}\right|$ as a function of e-folds for two different momenta during the dilaton-driven inflation and the graceful exit. We choose the initial value of dilaton to be $\phi=-140$, in which case one has sufficient amount of inflation $(N>60)$. We also plot the evolution of $\dot{\phi}$ and $H$, which are nearly constant during the string phase. Due to the presence of loop corrections, singularity avoidance is realized around $N=60$.

During the string phase with constant $\dot{\phi}$ and $H$, one easily finds that $a \propto(-\eta)^{-1}$ and $Q \propto e^{-\phi} \propto(-\eta)^{\dot{\phi}_{f}} / H_{f}$, which yields $\alpha=-1+\dot{\phi}_{f} /\left(2 H_{f}\right)$. Therefore, when $s$ is positive constant, the spectral tilt of the large scale curvature perturbation is $n-1=3-\left|3-\dot{\phi}_{f} / H_{f}\right| \simeq$ 2.26. It was shown in ref. [12] that the ratio $\dot{\phi}_{f} / H_{f}$ is required to range in the region $2<\dot{\phi}_{f} / H_{f}<3$ for the successful graceful exit in the presence of other forms of $\alpha^{\prime}$ corrections such as $G^{\mu \nu} \partial_{\mu} \phi \partial_{\nu} \phi$ and $\square \phi\left(\partial_{\mu} \phi\right)^{2}$. While it is possible to have sufficient amount of inflation $(N>$ $60)$ in these scenarios, the spectral indices are constrained to be $3<n<4$ for the constant positive $s$, implying the difficulty to generate the flat spectra sourced by the dilaton fluctuation.

In the present model with fixed points $\dot{\phi}_{f} \simeq 1.40$ and $H_{f} \simeq 0.62, s$ changes sign during a short transition from the dilaton-driven to the string phase, after which $s$ is approximately negative constant until the graceful exit. In this case the solution for eq. (2.8) can be written in the form

$$
\Psi_{k}=\sqrt{-\eta}\left[c_{1} I_{\nu}(x)+c_{2} K_{\nu}(x)\right],
$$

where $x=-\sqrt{|s|} k \eta$. Here $I_{\nu}$ and $K_{\nu}$ are modified Bessel functions, whose asymptotic solutions are $I_{\nu} \propto k^{\nu}$, $K_{\nu} \propto k^{-\nu}$ for $x \rightarrow 0$, and $I_{\nu} \sim e^{x} / \sqrt{2 \pi x}, K_{\nu} \sim$ $\sqrt{\pi /(2 x)} e^{-x}$ for $x \rightarrow \infty$. Therefore in the large scale limit $\left(\left|s k^{2}\right| \ll\left|z^{\prime \prime} / z\right|\right)$, one reproduces the spectrum $P_{\mathcal{R}} \propto k^{\dot{\phi}_{f}} / H_{f}$ as in the case of the constant positive $s$. For the modes which are inside the horizon, the curvature perturbation exhibit exponential increase, after which $\mathcal{R}_{k}$ is frozen as is found in Fig. 2. Since $\alpha=-1+\dot{\phi}_{f} /\left(2 H_{f}\right)$ is smaller than $1 / 2$, the curvature perturbation is frozen after the horizon crossing. This conservation is similar to the standard slow-roll inflationary scenarios, but the spectrum of the density perturbation is highly blue-tilted due to the enhancement of small scale fluctuations inside the horizon. It is also worth mentioning that at the graceful exit $\mathcal{R}_{k}$ remains finite together with the gravitational potential (see Fig. 2).

\section{SUMMARY AND DISCUSSIONS}

We shall summarize the density perturbation spectra in Fig. 3. The quantity $z=a \sqrt{Q}$ is an important one to determine the spectra and the evolution of curvature perturbations. When $z \propto(-\eta)^{\alpha}$ and the quantity $s$ is timeindependent, the spectral tilts in the large scale limit $\left(\left|s k^{2}\right| \ll\left|z^{\prime \prime}\right| z \mid\right)$ are given by eq. (2.13), which are plotted by the dotted line in Fig. 3. We also show the region where the curvature perturbation is enhanced on superhorizon scales $(\alpha \geq 1 / 2)$.

The simplest version of the pre-big bang scenario corresponds to the case of $\alpha=1 / 2$ and $n-1=3$. In the Ekpyrotic Universe or the dilaton-driven inflation with higherorder corrections, the spectral tilts are $2<n-1 \leq 3$ with $0<\alpha \leq 1 / 2$. In the modulus-driven inflation with a Gauss-Bonnet term, one has $\alpha=1$ and $n-1=7 / 3$. 
In this case the spectral index is not on the line of $n-1=3-|1-2 \alpha|$ due to the fact that $s$ is the timevarying function. In addition the curvature perturbation exhibits parametric amplification on superhorizon scales since $\alpha$ is larger than $1 / 2$.

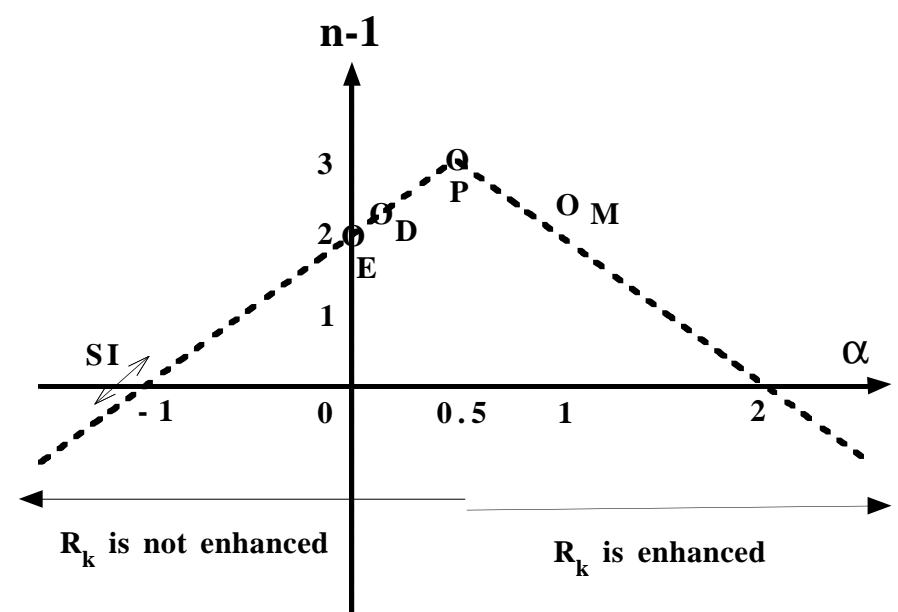

FIG. 3. The spectral tilts in string-inspired cosmological models. "P, E, M, D" correspond to the case of the pre-big-bang scenario, Ekpyrotic Universe, modulus-driven inflation with a Gauss-Bonnet term, and dilaton-driven inflation with higher-order corrections, respectively. "SI" denotes the standard slow-roll inflation.

In string-inspired models considered in this work, we obtain blue spectra with $n>3$. In order to generate the scale-invariant spectra ( $n \simeq 1$ ), the background evolution is required to be $\alpha \simeq-1$ or $\alpha \simeq 2$. The standard slow-roll inflation corresponds to the former case with conserved curvature perturbations after horizon crossing, while the latter one is the case where $\mathcal{R}$ is enhanced on superhorizon scales. It is certainly of interest to investigate whether there exist some models motivated by string theory which lead to the spectral indices with $|n-1| \lesssim 1$.

Even if the single-field string-inspired scenarios have some difficulty to generate the flat spectra, the quantum fluctuation of a light scalar field such as the axion may originate the large-scale curvature perturbations [27]. In fact it was recently claimed in ref. 28] that scale-invariant curvature perturbations may be obtained after inflation if a scalar field called "curvaton" produces an almost flat spectrum of isocurvature perturbations due to a light mass during inflation (see also refs. 29,30]). The applications of this idea to the string-inspired models are left to future work.

\section{ACKOWLEDGMENTS}

The author thanks Bruce Bassett, Robert Brandenberger, Cyril Cartier, Alexei Starobinsky, and Jun'ichi Yokoyama for useful discussions. He is also thankful for financial support from the JSPS (No. 04942).
[1] J. E. Lidsey, D. Wands and E. J. Copeland, Phys. Rep. 337, 343 (2000).

[2] G. Veneziano, Phys. Lett. B 265, 287 (1991); M. Gasperini and G. Veneziano, Astropart. Phys. 1, 317 (1993); Mod. Phys. Lett. A 8, 3701 (1993).

[3] M. Gasperini and G. Veneziano, Phys. Rev. D 50, 2519 (1994).

[4] R. Brustein and G. Veneziano, Phys. Lett. B 329, 429 (1994).

[5] R. Easther, K. Maeda, and D. Wands, Phys. Rev. D 53, 4247 (1996).

[6] I. Antoniadis, J. Rizos, and K. Tamvakis, Nucl. Phys. B415, 497 (1994); J. Rizos and K. Tamvakis, Phys. Lett. B 326, 57 (1994).

[7] R. Easther and K. Maeda, Phys. Rev. D 54, 7252 (1996).

[8] S. Kawai and J. Soda, Phys. Rev. D 59, 063506 (1999); H. Yajima, K. Maeda, and H. Ohkubo, Phys. Rev. D 62, 024020 (2000).

[9] M. Gasperini, M. Maggiore, and G. Veneziano, Nucl. Phys. 494, 315 (1997).

[10] R. Brustein and R. Madden, Phys. Rev. D 57, 712 (1998).

[11] S. Foffa, M .Maggiore, and R. Sturani, Nucl. Phys. 552, 395 (1999).

[12] C. Cartier, E. J. Copeland, and R. Madden, JHEP 01, 035 (2000); C. Cartier, E. J. Copeland, and M. Gasperini, Nucl. Phys. B607, 406 (2001).

[13] R. H. Brandenberger, R. Easther, and J. Maia, JHEP 9808, 007 (1998).

[14] J. Khoury, B. A. Ovrut, P. J. Steinhardt, and N. Turok, hep-th/0103239.

[15] J. Khoury, B. A. Ovrut, P. J. Steinhardt, and N. Turok, hep-th/0109050.

[16] R. Brustein et al., Phys. Rev. D 51, 6744 (1995).

[17] J. Hwang and H. Hoh, Phys. Rev. D 61, 043511 (2000).

[18] C. Cartier, J. Hwang and E. J. Copeland, Phys. Rev. D 64, 103504 (2001).

[19] S. M. Leach, M. Sasaki, D. Wands, and A. R. Liddle, Phys. Rev. D 64, 023512 (2001).

[20] D. H. Lyth, hep-th/0106153.

[21] R. H. Brandenberger and F. Finelli, hep-th/0109004.

[22] J. Hwang, astro-ph/0106197.

[23] D. H. Lyth, hep-ph/0110007.

[24] S. Kawai and J. Soda, Phys. Lett. B 460, 41 (1999).

[25] A. A. Starobinsky, S. Tsujikawa, and J. Yokoyama, Nucl. Phys. B610, 383 (2001).

[26] P. Peter and N. Pinto-Neto, gr-qc/0109038.

[27] E. J. Copeland, R. Easther, and D. Wands, Phys. Rev. D 56, 874 (1997).

[28] D. H. Lyth and D. Wands, hep-ph/0110002.

[29] E. Enqvist and M. S. Sloth, hep-ph/0109214.

[30] T. Moroi and T. Takahashi, Phys. Lett. B 522, 215 (2001). 